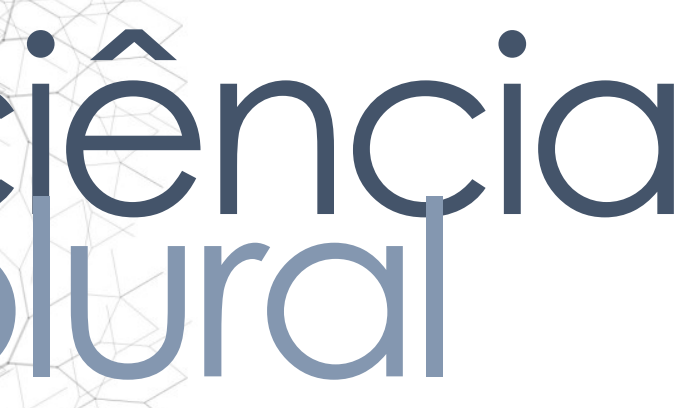

\title{
ONFALITE: UMA REALIDADE DE SAÚDE PÚBLICA EM ANGOLA
}

Onfalite: a public health reality in Angola

Onfalite : una realidad de salud pública en Angola

Elsy Tavares - Investigadora do CEMRI, G.I. Saúde, Cultura e Desenvolvimento, CEMRI/Uab • Doutoranda da Universidade Aberta de Lisboa -Portugal • E-mail: el.tavar@gmail.com

Natália Ramos - Professora Associada da Universidade Aberta de Lisboa/UAb -Portugal • Coordenadora Científica do Centro de Estudos das Migrações e das Relações Interculturais/CEMRI - Investigadora Responsável do G.I. Saúde, Cultura e Desenvolvimento, CEMRI/Uab E-mail: Maria.Ramos@uab.pt

Autora correspondente:

Elsy Tavares • E-mail: el.tavar@gmail.com 


\section{RESUMO}

Introdução: O presente estudo apresenta uma grave questão de saúde pública em Luanda, Angola, relacionada com situações de internamento e morte de recémnascidos por onfalite que o país enfrenta. Objetivos: descrever a realidade de um hospital público geral na cidade de Luanda, relativamente a este problema; identificar as principais causas de onfalites no contexto Angolano; descrever os cuidados corretos com o coto umbilical; evidenciar as implicações sociais, culturais e de saúde nos cuidados ao recém-nascido. Método: estudo descritivo, exploratório e transversal. Foram analisados os processos de internamento de um serviço de pediatria de um hospital público com situações de internamento por onfalite, entre janeiro de 2017 e junho de 2018. Resultados: no período em estudo, foram analisados 182 processos de internamento neste hospital com registos de onfalites no recém-nascido. Destes internamentos, 26 culminaram em óbito. Dos 156 recém-nascidos internados, 153 tiveram alta para o domicílio, 2 tiveram transferência para o hospital pediátrico e houve um registo de fuga. Conclusão: os resultados obtidos demonstram que as mães e os recém-nascidos angolanos permanecem vulneráveis no que se refere aos cuidados ao coto umbilical, o que se traduz pelo elevado número de onfalites registadas. Tornase, assim, prioritária a formação dos profissionais de saúde nesse domínio, de modo a que as intervenções sejam efetivas e de qualidade, no sentido de diminuir este flagelo de saúde pública neste país.

Palavras Chave: Cordão umbilical; Recém-nascido; Mortalidade infantil; Cuidados infantis; Cultura.

\section{ABSTRACT}

Introduction: This study presents a serious public health issue in Luanda, Angola, related to situations of hospitalization and death of newborns due to omphalitis that the country faces. Objectives: to describe the reality of a general public hospital in the city of Luanda, regarding this problem; identify the main causes of omphalitis in the Angolan context; describe the correct care with the umbilical stump; evidence the social, cultural and health implications of newborn care. Method: descriptive, exploratory and cross-sectional study. The hospitalization processes of a pediatric service of a public hospital with situations of hospitalization for omphalitis between January 2017 and June 2018 were analyzed. Results: during the study period, 182 hospitalization processes were analyzed in this hospital with records of omphalitis in the newborn. Of these hospitalizations, 26 culminated in death. Of the 156 newborns admitted, 153 were discharged to the home, 2 were transferred to the pediatric hospital and there was a record of a leak. Conclusion: the results obtained demonstrate that Angolan mothers and newborns remain vulnerable with regard to the care of the umbilical stump, which translates into the high number of registered omphalitis. Thus, training of health professionals in this field becomes a priority, so that terventions are effective and of quality, in order to reduce this scourge of public lth in this country.

words: Umbilical cord; Newborn; Child mortality; Child care; Culture. 


\section{RESUMEN}

Introducción: El presente estudio presenta un grave problema de salud pública en Luanda, Angola, relacionado con situaciones de hospitalización y muerte de recién nacidos debido a la onfalitis que enfrenta el país. Objetivos: describir la realidad de un hospital público general en la ciudad de Luanda, con respecto a este problema; identificar las principales causas de onfalitis en el contexto angoleño; describa el cuidado correcto con el muñón umbilical; evidencia las implicaciones sociales, culturales y de salud del cuidado del recién nacido. Método: estudio descriptivo, exploratorio y transversal. Se analizaron los procesos hospitalarios de un servicio pediátrico de un hospital público con situaciones de hospitalización por onfalitis entre enero de 2017 y junio de 2018. Resultados: en el período en estudio, se analizaron 182 procesos de hospitalización en este hospital con registros de onfalitis en el recién nacido De estas hospitalizaciones, 26 culminaron en la muerte. De los 156 recién nacidos admitidos, 153 fueron dados de alta a la casa, 2 fueron trasladados al hospital pediátrico y hubo un registro de una fuga. Conclusión: los resultados obtenidos demuestran que las madres y los recién nacidos angoleños siguen siendo vulnerables con respecto al cuidado del muñón umbilical, lo que se traduce en un alto número de onfalitis registrada. Por lo tanto, la capacitación de profesionales de la salud en este campo se convierte en una prioridad, para que las intervenciones sean efectivas y de calidad, a fin de reducir este flagelo de la salud pública en este país.

Palabras clave: Cordón umbilical; Recién nacido; Mortalidad infantil; Cuidado de niños; Cultura. 


\section{Introdução}

Em Angola a área da saúde materno-infantil é uma preocupação espelhada no Plano Nacional de Desenvolvimento Sanitário de Angola 20122025 (PNDS), no Programa de Prevenção e Luta contra as Doenças, que inclui no projeto 18:"a prestação dos cuidados de saúde para a sobrevivência materna, neonatal e infantil", sendo uma das metas, a redução em $50 \%$ das taxas de mortalidade materna e infantil 1 .

O período neonatal, que corresponde aos primeiros 28 dias de vida, é um período crítico, revestido de enorme vulnerabilidade e risco de morte, sendo que em Angola a mortalidade neonatal é estimada em 42/1000 nascidos vivos, cujas principais causas são a asfixia, a prematuridade e a sepse ${ }^{1}$.

De acordo com a World Health Organization² (2016), a nível mundial, cerca de 5,9 milhões de crianças com menos de cinco anos morrem anualmente, estimando-se que $45 \%$ dessas mortes ocorram no período neonatal. A mesma fonte refere que, anualmente, em todo o mundo, cerca de 2,8 milhões de crianças morrem durante o primeiro mês, a maioria em países em desenvolvimento. Em 2016, morreram, por dia, 15.000 crianças menores de 5 anos, das quais $46 \%$, isto é, 7.000 crianças, morreram nos primeiros 28 dias de vida ${ }^{3}$.

$\mathrm{O}$ presente estudo baseia-se nos seguintes objetivos: descrever a realidade de um hospital público geral na cidade de Luanda, relativamente à onfalite; identificar as principais causas de onfalites no contexto Angolano; descrever os cuidados corretos com o coto umbilical; evidenciar as implicações sociais, culturais e de saúde nos cuidados ao recém-nascido.

\section{Referêncial teórico}

fuidados ao recém-nascido e Onfalite em Luanda, Angola

O nascimento é um acontecimento que mobiliza toda a família em torno s cuidados dedicados ao novo ser. Nesse contexto familiar, as mães e avós presença constante e participam com suas experiências, assumindo os dados prestados ao recém-nascido, tais como o primeiro banho, a toilete e os dados com o coto umbilical ${ }^{4}$. 
Em Luanda, é frequente as mães, mesmo sendo acompanhadas numa unidade de saúde, optarem por condutas de aplicação de soluções caseiras e inadequadas, adotadas como primeira escolha nos cuidados realizados ao coto umbilical, apesar de cientificamente comprovados a sua ineficácia e os danos para a saúde do recém-nascido. Algumas das práticas frequentes no cuidado ao coto umbilical realizadas pelas mães em Luanda, e transmitidas de geração em geração, são: enfaixar o coto, colocar moedas, usar banha de galinha, folhas queimadas, cinza, sal, barata queimada, óleo de palma, azeite, leite materno, pó de café, fezes e aranha ${ }^{4}$.

O coto umbilical do recém-nascido implica a necessidade de cuidados diários. Para além da limpeza correta deste, o profissional de saúde deve orientar para as medidas de higiene das mãos, de quem vai manipular o coto e para a troca frequente de fraldas, colocando uma compressa limpa para cobrir o coto. Deve ser feita, igualmente uma dobra na fralda, a qual deve ser colocada abaixo do coto umbilical para evitar irritação e a proliferação de microrganismos ${ }^{4}$.

As assimetrias verificadas nas comunidades devido às grandes desigualdades sociais e de oportunidades em todo o território angolano, provocam movimentos migratórios internos para os centros urbanos, principalmente Luanda, com sobrecarga dos serviços públicos disponíveis. Compreende-se, assim, a multiculturalidade existente nesta cidade, bem como o enorme desafio no que diz respeito a questões sociais e de saúde, sendo que a elevada concentração de migrantes na cidade exprime a grande diversidade de traços culturais, de práticas e costumes, de estilos de vida e de identidades ${ }^{5}$.

Estas mães vivem frequentemente em contexto socioeconómico precário, de superpopulação e com falta de condições de conforto e higiene, o que coloca em risco a saúde do recém-nascido, com implicações para a sua sobrevivência e em-estar geral. No processo de aculturação, as mães poderão ter dificuldade seleção entre os aspectos a manter e abandonar da sua cultura de origem, por vezes contrastam com o efeito da cultura ocidental nos cuidados ao m-nascido ${ }^{5}$. 
A incidência de onfalite em recém-nascidos em países desenvolvidos é de $0,7 \%$, subindo para $2,7 \%$ nos países em desenvolvimento 6 . À semelhança de Angola, de acordo com Linhares et al. ${ }^{7}$, num estudo realizado na Bahia (Brasil), a onfalite deriva da utilização de substâncias como pó de pena de galinha e pó de café.

Em 2011, verificou-se em Angola 96 mortes em crianças com menos de um ano a cada mil nascimentos. Neste cenário, entre 1990 e 2011, Angola apresentou uma das menores taxas de redução da mortalidade infantil de toda a África Subsaariana, com $2,1 \%$ ao ano ${ }^{8}$.

No ano de 2012, a taxa de mortalidade neonatal em Angola foi de 51,1\% . No mesmo ano, no serviço de neonatologia de uma maternidade de Luanda, em cada 10 crianças internadas, quatro morreram antes de completar o $1^{\circ}$ mês de vida, das quais aproximadamente $30 \%$ morreram na primeira semana de vida 9 .

Em 2015, segundo Wateraid 10, Angola foi considerado o local mais perigoso do mundo para se ter um bebê, com as infecções a representarem quase $30 \%$ de todas as mortes neonatais. Com um número de mortes neonatais a atingir os 42.625, destes, o número de recém-nascidos perdidos devido à sepse/meningite/tétano foi de 9.053, sendo a percentagem de mortes neonatais de 21,2 e a probabilidade de uma mãe perder o bebê devido a sepse de 1 em 1710 .

Em Angola o problema das onfalites também é mencionado nos meios de comunicação social. Por exemplo, em declarações à Agência Angola Press ${ }^{11}$, uma médica do Hospital Pediátrico de Luanda manifestou a preocupação com a situação, referindo que têm sido muitos os casos de onfalites recebidos no hospital e que muitas mães curam o umbigo dos bebês com sal e pó de esteira queimada, levando-os a contrair infecções graves e à morte. Referiu, ainda, que maioria das mães leva os filhos ao hospital já com a doença em estado avançado, abando alguns por morrer ${ }^{11}$. Uma outra médica do serviço de urgência do ospital Pediátrico de Luanda, referiu que, entre janeiro e março de 2018, foram iagnosticados um total de 309 recém-nascidos com problemas de sepse onatal, tendo-se registrado um total de 67 mortes, assim distribuídas: em 
janeiro, 74 recém-nascidos internados e 15 óbitos; em fevereiro, dos 85 internamentos, verificaram-se 30 óbitos; e em março, observou-se o maior número de registros de internamento por septicemia, ou seja, 150 , sendo que destes resultaram 22 óbitos ${ }^{12}$.

Os profissionais de saúde devem compreender o contexto social em que as mães se inserem, e realizar uma vigilância continua durante o período neonatal, a fim de detectar possíveis problemas, minimizar os riscos e melhorar a qualidade de vida do recém-nascido, de modo a garantir o seu crescimento e desenvolvimento saudável ${ }^{13}$.

\section{Evolução dos cuidados ao coto umbilical}

A prática do cuidar é seguramente o mais antigo procedimento da história do mundo. Cuidar é inerente à sobrevivência de todos os seres vivos, garantindo a continuidade da vida da espécie humana ${ }^{14}$.

O período puerperal, que pode decorrer entre 6 a 8 semanas após o parto, é revestido de mitos, crenças, tabus, costumes e medos, nos quais os cuidados ao recém-nascido são permeados por práticas culturais, transmitidas através de gerações, que se refletem também nos cuidados ao coto umbilical. Algumas mães referem-se a este como algo de intocável, que representa um elevado perigo para a saúde da criança ${ }^{5}$. As mães, principalmente as primíparas, sentem-se inseguras em relação ao manuseio e à queda do coto, delegando por vezes o seu cuidado a outras pessoas, que não são o foco da atenção das orientações dos profissionais de saúde ${ }^{14}$.

A teoria da diversidade e universalidade do cuidado cultural de Madeleine Leinninger baseia-se na universalidade e diversidade do cuidado sob a influência cultural do meio envolvente com a interpretação dos diversos significados culturais do cuidar. Compete ao profissional de saúde, encarar com ensibilidade os saberes associados às práticas dos cuidados. Ao conhecer a periência familiar e cultural do cuidado ao recém-nascido, pode-se gramar cuidados coerentes e em conjunto com a família. Assim, para que o lado humano seja significativo e terapêutico, o profissional precisa de se 
adequar e respeitar os valores culturais, convicções e expectativas do paciente, mas garantindo sempre a qualidade dos cuidados ${ }^{14}$.

O cordão umbilical possui um tecido em processo de desvitalização, o que o torna um meio de cultura propício ao desenvolvimento de bactérias, além de possuir vasos que permitem o acesso direto à corrente sanguínea. Após o nascimento, o cordão umbilical é clampado e cortado, e passa a ser chamado de coto umbilical. O processo de mumificação do coto umbilical ocorre perto do terceiro ou quarto dia, e o seu desprendimento da parede abdominal ocorre entre o quarto e o oitavo dia de vida, podendo estender-se até 14 ou 15 dias depois do nascimento ${ }^{15}$.

O tétano neonatal é uma infeção causada pelo Clostridium Tetani e ocorre pela contaminação do coto umbilical do recém-nascido através de instrumentos não esterilizados ou por cuidados inadequados para a sua cicatrização ${ }^{16}$. Os fatores de risco mais presentes, nos problemas com o coto umbilical são o fraco acompanhamento do recém-nascido e da puérpera pelos serviços de saúde, déficit nos cuidados de higiene, práticas incorretas no cuidado ao coto umbilical, baixo nível de escolaridade das mães e baixo nível socioeconômico e carências sociais e de saúde 16 .

A evolução histórica dos cuidados ao recém-nascido demonstra que, no passado, estes eram prestados por pessoas não qualificadas, que proporcionavam cuidados não diferenciados, de forma empírica e baseados no saber do senso comum, sendo frequente a utilização de solutos como óleos, preparações caseiras e colostro, práticas que ainda se constatam em Angola. No entanto, na atualidade, estes cuidados são, em geral, garantidos por profissionais qualificados, que proporcionam cuidados de qualidade, baseados na evidência científica, principal requisito para cuidados de saúde de qualidade

Com o evoluir dos tempos, surgiram medicamentos para aplicação ica no coto, como o corante triplo, a iodopovidona, o álcool a 70ª rehexidina e, nas últimas décadas, assistiu-se à utilização da técnica dry care, se traduz na limpeza do coto com água e sabão e em mantê-lo limpo e seco, 
sem aplicar qualquer tipo de antisséptico ou desinfetante. Deste modo, ao longo dos anos, várias técnicas e produtos foram aplicados no coto umbilical com o objetivo de higienizar corretamente, promover a desidratação, mumificação e queda, ou seja, acelerar o processo de isquemia e reduzir a ocorrência de infeção ${ }^{17}$.

Segundo Correia \& Pires ${ }^{18}$, a OMS, desde 1998, recomenda a técnica $d r y$ care nos cuidados ao cordão umbilical do recém-nascido. No entanto, orienta também para o uso de antissépticos no coto umbilical em países em desenvolvimento, onde existem hábitos precários de higiene, partos em casa e elevada taxa de incidência de infecção ou mortalidade neonatal. Assim, a clorehexidina 0,5\% deve ser o agente preferido, e na sua ausência, ou por ser economicamente mais acessível, deve-se utilizar o álcool etílico a 70\%, com aplicação entre 7 a 10 dias, mantendo o coto exposto até que evapore.

\section{Cuidados culturalmente competentes}

Na sociedade angolana, tal como na maioria das sociedades, coexistem vários métodos terapêuticos, crenças e práticas de saúde, baseados em princípios e modelos originários de culturas diferentes, coexistindo um sistema de saúde oficial com modelos alternativos ${ }^{19}$.

A competência (inter)cultural nos profissionais de saúde permite capacitar e dar uma resposta efetiva às necessidades das mães e cuidadores, considerando os contextos culturais, sociais e de saúde em questão, através do estabelecimento da relação terapêutica e negociação. Isto implica, para o profissional de saúde, formação na área da interculturalidade, de modo a conseguir dar resposta à diversidade cultural e individual, com uma abordagem humanizada, promovendo a equidade e a qualidade nos cuidados de saúde, a articulação das mães e comunidade com as unidades de saúde ${ }^{19}$.

O desconhecimento das consequências acerca das práticas sociais e turais às quais as mães recorrem e da forma como as mesmas realizam o idado e o justificam, pode criar barreiras nos cuidados prestados pelos fissionais de saúde e comprometer o sucesso das intervenções ${ }^{19}$. 
A prestação de cuidados culturalmente competentes é uma emergência na promoção da saúde e bem-estar das mães e recém-nascidos. Implica investir, a nível da formação sólida, sobre a complexidade de fatores explicativos e intervenientes nos comportamentos de saúde, e desenvolvimento de competências profissionais, comunicacionais, interculturais, relacionais, de cidadania e consciencialização cultural ${ }^{19,20}$.

A comunicação em contexto de saúde tem de estar adaptada às capacidades cognitivas, ao nível cultural e educacional, às representações e crenças de saúde, às necessidades individuais, emocionais, sociais, culturais e linguísticas das mães, família e comunidade, com o objetivo de facilitar a educação para a saúde e promover cuidados de saúde culturalmente competentes ${ }^{20,21}$.

O empoderamento, ou seja, o processo de aquisição de conhecimentos e desenvolvimento de competências, permite a participação ativa na tomada de decisão efetiva e a autorresponsabilização dos cuidados realizados ao coto umbilical do recém-nascido. A aquisição de competências permite a estas mães defenderem as práticas aprendidas e transmitidas pelo profissional de saúde, quando são confrontadas com práticas culturais e tradicionais impostas pelo meio familiar ou comunitário, ou seja, através da educação e informação, as mães ficam capacitadas para agir sobre os determinantes de saúde ${ }^{22}$.

Portanto, torna-se prioritária a assistência às mães e a formação dos profissionais de saúde nesse domínio, de modo a que as suas intervenções junto a elas e aos recém-nascidos sejam efetivas e de qualidade, no sentido de prevenir a realidade preocupante do elevado número de onfalites e diminuir práticas prejudiciais à saúde da criança que ainda hoje se mantêm em Angola 23.

\section{letodologia}

Trata-se de um estudo descritivo, exploratório e transversal. Procedeuanálise dos dados constantes dos processos referentes ao internamento de m-nascidos por onfalite no serviço de pediatria, do Hospital Municipal de 


\section{ciênciála
pural}

Luanda, entre janeiro de 2017 e junho de 2018. Utilizou-se a técnica de amostragem não probabilística por conveniência. Foram respeitados os princípios éticos da colheita e tratamento de dados, sendo os mesmos utilizados apenas para o estudo e não havendo a divulgação da identidade dos recémnascidos e/ou cuidadores. Como critérios de inclusão, foram considerados todos os recém-nascidos internados por onfalite, ou septicemia por onfalite, no serviço de pediatria deste hospital no período referido. Foi realizada análise documental através de busca bibliográfica especializada e relevante sobre o tema em investigação, permitindo o aprofundamento, descrição, análise e discussão dos resultados.

\section{Resultados}

No período em estudo, foram analisados 182 processos de internamento do serviço de pediatria do Hospital Municipal de Luanda, com registros de onfalites em recém-nascidos. Destes 182 internamentos, 26 culminaram em óbito do recém-nascido.

Internamentos de recém-nascidos por onfalite sem ocorrência de óbito

Verificou-se um total de 156 internamentos, dos quais 54 ocorreram em 2017 e 101 no primeiro semestre de 2018, havendo um processo sem registro de data. Em relação ao sexo do recém-nascido, registraram-se 74 do sexo masculino e 78 do sexo feminino, sendo que quatro não apresentam informação relativa ao sexo. A proveniência da criança, em 149 situações, é do domicílio, e nas restantes não existe registro. Quanto ao diagnóstico do internamento, em 79 situações, a causa foi onfalite, e nas outras 77 deveu-se a septicemia, cuja causa inicial foi a onfalite. Em todos os dados de internamento verificou-se que a antibioticoterapia constou do tratamento prescrito. Em relação aos cuidados realizados ao coto umbilical em casa, não consta quem o praticou. No total de internamentos, percebeu-se um caso de fuga com o recém-nascido, duas ransferências para um hospital pediátrico em Luanda, tendo os restantes 153 ém-nascidos tido alta para o domicílio. Os dados relativos à idade (em dias), recém-nascido à data de admissão, a quem o leva ao hospital, aos produtos 
aplicados no coto umbilical e aos dias de internamento, podem observar-se na Tabela 1.

Tabela 1: Idade (em dias) à data de admissão, quem leva ao hospital, produtos aplicados no coto umbilical e dias de internamento, nos recém-nascidos internados por onfalite sem ocorrência de óbito. Luanda, 2019.

\begin{tabular}{|c|c|}
\hline VARIÁVEL & $\mathbf{n}$ \\
\hline Idade do recém-nascido (RN) em dias, à data de admissão & $\mathbf{N}^{0}$ de $\mathbf{R N}$ \\
\hline 1 & 24 \\
\hline 2 & 20 \\
\hline 3 & 20 \\
\hline 7 & 19 \\
\hline 28 & 1 \\
\hline Sem registro & 72 \\
\hline Quem leva a criança ao hospital & $\mathbf{N}^{0}$ de $\mathbf{R N}$ \\
\hline Mãe & 137 \\
\hline Pai e mãe & 6 \\
\hline Pai & 5 \\
\hline Outros familiares & 3 \\
\hline Sem registro & 5 \\
\hline Produtos aplicados no coto umbilical & $\mathbf{N}^{0}$ de $\mathbf{R N}$ \\
\hline Sal & 3 \\
\hline Óleo de palma & 1 \\
\hline Pó (sem especificar) & 1 \\
\hline Químicos (sem especificar) & 1 \\
\hline Sem registro & 150 \\
\hline Dias de internamento & $\mathbf{N}^{0}$ de $\mathbf{R N}$ \\
\hline 1 & 17 \\
\hline 2 & 30 \\
\hline 3 & 32 \\
\hline 24 & 1 \\
\hline Sem registro & 76 \\
\hline
\end{tabular}

Fonte: Elaboração das autoras.

Internamentos de recém-nascidos por onfalite com ocorrência de óbito

Verificou-se um total de 26 óbitos de recém-nascidos. Destes, 21 ocorreram em 2017 e 5 no primeiro semestre de 2018. Quanto ao sexo dos recémnascidos falecidos, 18 eram do sexo feminino e 8 do sexo masculino. Em 16 situações, a onfalite foi a causa de morte, e em 10 situações, septicemia, tendo como a causa primária a onfalite. Em todos os internamentos verificou-se a rescrição de antibioterapia como tratamento. Os dados relativos à idade (em as) do recém-nascido à data de admissão, a quem leva ao hospital, aos dutos aplicados no coto umbilical e aos dias de internamento, podem 
observar-se na tabela 2. Importante ressaltar que a maioria dos dias de internamento se situou entre os 0 e 1 dias e também a ausência frequente de registros.

Tabela 2: Idade (em dias) à data de admissão, quem leva ao hospital, produtos aplicados no coto umbilical e dias de internamento, nos recém-nascidos internados por onfalite com ocorrência de óbito. (Luanda, 2019).

\begin{tabular}{|c|c|}
\hline VARIÁVEL & $\mathbf{n}$ \\
\hline Idade do recém-nascido (RN) em dias, à data de admissão & $\mathbf{N}^{0}$ de $\mathbf{R N}$ \\
\hline 1 & 2 \\
\hline 3 & 4 \\
\hline 4 & 4 \\
\hline 6 & 4 \\
\hline 10 & 11 \\
\hline 21 & 1 \\
\hline Quem leva a criança ao hospital & $\mathbf{N}^{0}$ de $\mathbf{R N}$ \\
\hline Mãe & 24 \\
\hline Sem registro & 2 \\
\hline Produtos aplicados no coto umbilical & $\mathbf{N}^{0}$ de $\mathbf{R N}$ \\
\hline Óleo de palma & 2 \\
\hline Sem registro & 24 \\
\hline Dias de internamento dos RN falecidos & $\mathbf{N}^{0}$ de $\mathbf{R N}$ \\
\hline 0 & 10 \\
\hline 1 & 8 \\
\hline 2 & 3 \\
\hline 3 & 1 \\
\hline 5 & 1 \\
\hline Sem registro & 3 \\
\hline
\end{tabular}

Fonte: Elaboração das autoras.

\section{Discussão}

Constatou-se que, em relação aos internamentos por onfalite, o número foi consideravelmente maior no primeiro semestre de 2018 com 101 internações, comparativamente ao ano de 2017, em que se registraram 54. Esta situação é larmante, se considerarmos que o número de hospitalizações num semestre é o dobro do ano anterior. Este fato alerta-nos para a necessidade urgente de intervenção dos profissionais de saúde através da educação das mães lativamente aos cuidados ao recém-nascido, concretamente ao coto umbilical. itas mães têm receio na sua manipulação e, enquanto não ocorre a sua da, recorrem a ajuda de terceiros para os seus cuidados. O cuidado é 
modelado pelo significado que as puérperas atribuem ao coto umbilical e pelas experiências e formas de cuidar expressas e adquiridas no meio intergeracional de pertença. A promoção dos cuidados deve iniciar-se nas consultas de vigilância pré-natal, continuando na maternidade e, posteriormente, na consulta do puerpério e em contexto domiciliar.

Contrariamente aos internamentos por estas causas ou ao número de óbitos em recém-nascidos por onfalite ou septicemia derivada de onfalite, o número foi superior no ano de 2017 com 21 óbitos, quando comparado com o primeiro semestre de 2018, em que se registraram 5 óbitos. No entanto, os números são preocupantes se considerarmos que se referem a mortes de recémnascidos por uma causa evitável, alertando, assim, para a necessidade de intervenção urgente no combate a este problema de saúde pública. Apesar de resultar de práticas incorretas, a onfalite pode ser facilmente tratada, quando precocemente identificada. As suas consequências podem ser muito graves, devido à rápida disseminação hematogênica e à sua extensão para outros órgãos. Carvalho, Markus, Abaggae, Giraldi, \& Campos (2015)²5, afirmam que cerca de $30 \%$ das mortes neonatais decorrem de infeções, onde os débeis cuidados ao coto umbilical são reconhecidos como potenciais causas de onfalite e septicemia.

Anualmente, em média, em todo o mundo ocorrem 4 milhões de mortes neonatais, das quais 99\% são devido a infeções, sendo estas do coto umbilical, denominadas de onfalite, a principal causa da mortalidade neonatal ${ }^{17}$.

Na grande maioria das situações de internamento, é a mãe quem leva o recém-nascido ao hospital, o que nos leva a pensar que seja ela a principal cuidadora do recém-nascido no domicílio. Sendo assim, é principalmente junto desta que se devem concentrar os esforços para o empoderamento e capacitação para o cuidado ao coto umbilical.

A capacitação das mães e familiares no sentido de que obtenham não enas conhecimento correto sobre a forma de cuidar do coto umbilical, mas nsiderem igualmente a sua responsabilidade em participar e contribuir para saúde do recém-nascido, em geral, é fundamental. A intervenção do 
profissional de saúde passa por orientar, substituindo práticas culturalmente adquiridas que possam ser nocivas e apresentem riscos para a saúde e reforçar práticas saudáveis. É fundamental promover o diálogo e capacitar as mães, bem como supervisionar o cuidado por elas realizado, tornando possível a elaboração, em conjunto, de um plano de cuidados, baseado nas necessidades identificadas, nas dificuldades e limitações das mães/família e nos cuidados ao coto umbilical, para a eliminação dos indicadores de morbi-mortalidade neonatal 4,19. Os profissionais de saúde pública, têm assim, pela sua proximidade com a comunidade, a possibilidade de colaborar de forma ativa na promoção do exercício da cidadania e no empoderamento das mães.

O fato de haver processos nos quais a idade dos recém-nascidos e os dias de internamento não se encontravam cadastrados constitui uma limitação ao estudo e uma preocupação relativamente à colheita de dados, registros e atuação dos profissionais de saúde. Também pelas falhas importantes e incompletitudedos dados, fica a dúvida se não terão ocorrido situações de tétano neonatal associado a onfalite, ou se não foram registradas, podendo ser consideradas uma limitação do estudo. Não existe registro de informação relativamente aos cuidados praticados, assim como de quem os realiza no domicílio. No total dos óbitos (26), apenas existem dois registros dos cuidados praticados, e no total de internamentos sem óbito (156), apenas seis. Estes dados poderiam revelar informação importante no sentido de saber quem cuida do recém-nascido e quais os saberes e práticas culturais e tradicionais inerentes a esse cuidado, orientando assim, a intervenção do profissional de saúde. Os cuidados inadequados e precários ao coto umbilical são reconhecidos como potenciais causas de onfalite e septicemia.

Apesar do número elevado de internamentos, as lacunas nos registros de saúde são evidentes. Concordamos com Linhares (2011) ${ }^{7}$, tratar-se de um tema qual tem sido dado pouca relevância, tendo a falta completa desses dados nsequências, tanto ao nível da avaliação epidemiológica, quanto do hecimento estatístico das ocorrências. 
Deste modo, a ausência destes registros remete-nos para a necessidade de investimento na formação dos profissionais de saúde, quer no aprofundamento da colheita de dados e na qualidade dos registros realizados que podem condicionar ou promover o sucesso das intervenções em saúde. Um dado adequadamente anotado contribui para o aperfeiçoamento e continuidade dos cuidados de saúde, tornando a informação menos fragmentada e mais completa, o que permite respostas mais eficazes e favorecendo a avaliação das intervenções ${ }^{23}$.

Quanto às idades registradas dos recém-nascidos falecidos, estes tinham na sua maioria até 10 dias de vida. $\mathrm{O}$ mesmo se verifica em relação às idades dos recém-nascidos internados que não faleceram. Apesar de ocorrerem internamentos até ao $28^{\circ}$ dia de vida, a maioria dos internamentos ocorreu entre o primeiro e o sétimo dias de vida, correspondendo ao período em que ocorre a queda do coto umbilical, ou seja, no período-chave dos cuidados ao mesmo. Os resultados vão ao encontro dos dados da Organização Mundial da Saúde 2, a qual relata que nos primeiros dias após o nascimento ocorrem cerca de $50 \%$ de óbitos neonatais e 75\% durante a primeira semana de vida.

No que se refere aos óbitos dos recém-nascidos, os dias de internamento em que ocorrem variam entre 0 e 5 dias, acontecendo em sua maioria nos primeiros dias, o que nos leva a suspeitar que a situação do recém-nascido quando da admissão na unidade hospitalar já era grave.

Nos internamentos onde não se verifica o óbito do recém-nascido, constata-se que, apesar do maior número de dias de internamento se verificar entre dois e três dias, existem situações em que o internamento se prolonga até os 24 dias . O aumento dos dias de internação do recém-nascido implica não só consequências econômicas para o Estado e para a família, com reflexo a nível social, mas também afeta a relação mãe/filho e com o restante da família. Para além destes aspectos, a exposição do recém-nascido ao ambiente hospitalar e o umento dos dias de internamento traduz-se em riscos acrescidos para a sua úde, uma vez que, pelas características inerentes à idade, este se encontra is vulnerável. 


\section{ciêncíncia
puurl}

\section{Conclusões}

Para desenvolver estratégias de prevenção e de intervenção em saúde é necessário conhecer a realidade de uma comunidade. Assim, conhecer o modo de viver de uma dada população, bem como os seus comportamentos, permite uma intervenção de saúde dirigida e adequada às reais necessidades.

De acordo com os resultados obtidos, verifica-se que os recém-nascidos e as mães angolanas permanecem vulneráveis no que se refere aos cuidados ao coto umbilical do recém-nascido. Para a redução da mortalidade neonatal tornase prioritária a assistência às mães e a formação dos profissionais de saúde, de modo que as suas intervenções junto das mães e dos recém-nascidos sejam efetivas e de qualidade.

Da análise efetuada aos registros que constam dos processos do internamento de pediatria do hospital em análise, verificou-se a pouca precisão dos mesmos, não constando informações importantes como, por exemplo, quais os cuidados realizados ao coto umbilical no domicílio e por quem; quais as orientações dadas às mães para os cuidados ao coto umbilical na maternidade; quais os seus saberes e práticas relativamente a este cuidado; e quem orientou a realização deste cuidado.

No que se refere à onfalite no recém-nascido, tratando-se de um problema de saúde pública existente em Angola, passível de prevenção, é importante o conhecimento e compreensão dos saberes e práticas tradicionais familiares e a influência social e cultural inerentes no cuidado ao coto umbilical, bem como a melhoria das práticas de cuidado e das condições de vida das famílias, além da melhoria dos registros de saúde e dos serviços e cuidados de saúde. Este conhecimento permite a prevenção do problema e a qualidade dos cuidados baseado num diagnóstico preciso e concreto da situação, direcionando intervenção do profissional de saúde, que surge como competência e ponsabilidade dos profissionais e serviços de saúde, proporcionando a moção de saúde dos recém-nascidos e, consequentemente, da sociedade ra. 
Este estudo sensibiliza para o investimento na formação dos profissionais de saúde na prestação de cuidados de saúde culturalmente competentes e incentiva a atenção dos profissionais e gestores políticos para este problema, de modo que as intervenções e políticas sejam dirigidas e adequadas às reais necessidades das mães e dos cuidadores, favorecendo assim a saúde e o desenvolvimento dos recém-nascidos.

O estudo possibilitou a mobilização de esforços por parte das entidades competentes no sentido de deslocarem meios e desenvolverem políticas públicas sociais e de saúde para o combate a este flagelo, contribuindo para atingir as metas preconizadas pelo Plano Nacional de Desenvolvimento Sanitário 2012-2025 e pelas Nações Unidas nos Objetivos de Desenvolvimento Sustentável - ODS.

Devido à escassez de estudos neste âmbito em Angola, sugere-se que se desenvolvam mais investigações, especialmente sobre os determinantes culturais e sociais e, sobre a forma como os próprios profissionais de saúde são influenciados pelos aspectos culturais nos cuidados ao recém-nascido, de modo a aumentar as bases que fundamentam e orientam as intervenções e políticas públicas no sentido da redução da onfalite em recém-nascidos em Angola e, consequentemente, a redução da morbidade e mortalidade neonatal.

\section{Referências}

1. Ministério da Saúde de Angola. Plano Nacional de Desenvolvimento Sanitário 2012-2025. (2). Luanda: Ministério da Saúde de Angola. 2012.

2. World Health Organization. Children: reducing mortality. 2016.

United nations interagency group for child mortality estimation. Levels \& Trends in Child Mortality. Report 2017. Estimates Developed by the UN Inter-agency Group for Child Mortality Estimation. 2017.

Ribeiro M.; Brandão M.A. Produção científica da enfermagem sobre coto umbilical. Revista Interdisciplinar Novafapi. 2011;4(3):54-59. 
5. Ramos N. A diversidade cultural na cidade: Problemas e Desafios. Rubim L. \& Miranda N. (org). Transversalidades da Cultura. Salvador: EDUFBA, 2008, p. 133-179.

6. López-Medina M.D.; Linares-Abad M.; López-Araque A.B.; López-Medina I.M. Cura a seco versus cura com clorexidina para prevenção da onfalite. Revisão sistemática com metanálise. Revista Latino-Americana. Enfermagem Artigo de Revisão. 2019; 27:3106.

7. Linhares L.; Silva L.; Rodrigues V.; Araújo R. Influência intergeracional no cuidado do coto umbilical do recém-nascido. Texto Contexto Enfermagem, Florianópolis. 2012; 21(4):828-36.

8. Simão R.; Gallo P. (2013). Mortes infantis em Cabinda, Angola: desafio para as políticas públicas de saúde. Revista Brasileira Epidemiologia. 2013; $16(4): 826-837$.

9. Cunha R.; Alves B.; Veiga G.; Adami F.; Carlesso J.; Figueiredo F. et al. Mortalidade neonatal em Luanda, Angola: o que pode ser feito para sua redução? Revista J Hum Growth. 2019; 29(2):161-168.

10. Wateraid. Começo saudável. Londres; 2015.

11. Agência Angola Press-Angop. Médica apela à cura de cordão umbilical com responsabilidade, Janeiro. 2009.

12. Jornal de Angola. Umbigos mal tratados levam a mortes. 27 de Abril. 2018.

13. Pereira M.; Souza D.; Souza A.; Ferreira T.; Andrade F. A assistência à saúde da criança: uma análise multidimensional dos serviços de saúde. Revista Ciência Plural. 2018; 4(3):57-68.

14. Rangel L.; Moreira M.; Jerónimo S. \& Ribeiro F. A prática do cuidado prestado pelas mulheres aos filhos no domicilio. Revista Enfermeria Global. 2007;10:1-9.

15. Lowdermilk L.; Perry E. Enfermagem na Maternidade. (7a ed.). Camarate: Lusodidacta; 2008.

16. Ministério da Saúde. Contribuição do sector de saúde para o plano nacional de desenvolvimento 2018-2022. Luanda: Angola; 2016.

Luís S.; Costa A. \& Casteleiro C. Boas práticas nos cuidados ao coto umbilical: um estudo de revisão. Millenium.2014;47:33-46. 
18. Correia T.; Pires C. Que técnica usar nos cuidados ao cordão umbilical do recém-nascido. Revista Da Associação Portuguesa Dos Enfermeiros Obstetras.2016. 1646-3625.

19. Ramos N. Mães e famílias entre culturas: saúde, desenvolvimento e cuidados interculturais. In Rocha M.; Ramos N.; Santos, S.; Costa M. D. (Org.). Seguridade social, interculturalidades e Desigualdades na contemporaneidade. Natal: EDUFRN, 2016, p. 229-269.

20. Ramos N. Migração, maternidade e saúde. Reportório, Teatro e Dança. Número Temático - Movimento, Criatividade e Cura. Salvador. 2012; 18 (1): 84-91.

21. Ramos N. Comunicação em saúde e interculturalidade - perspectivas teóricas, metodológicas e praticas. RECIIS - Revista Eletrónica de Comunicação Informação \& Inovação em Saúde. Rio deJaneiro.2012 ; 6(4): 1-19.

22. Leite R.B.; Pontes C.S. \& Pavão J.F. Cidadania para a saúde: o papel do cidadão na promoção da saúde. Lisboa: Universidade Católica Editora; 2015.

23.Ramos N.; Tavares E. Cuidados de mães Angolanas ao recém-nascido: Abordagem Intercultural. In H. Pereira, S. Monteiro, G. Esgalhado, A. Cunha, I. Leal (Ed.), (2020). Atas $13^{\circ}$ Congresso Nacional de Psicologia da Saúde - Melhorar o Bem-Estar Global através da Psicologia da Saúde.2020. p. 313- 322. Lisboa, ISPA.

24. Ferreira T.; Souza A.; Medeiros J.; Andrade F. Avaliação do atributo coordenação do cuidado em serviços de puericultura na atenção primária à saúde. Revista Ciência Plural. 2017; 3(1): 98-107.

25. Carvalho V.; Markus J.; Abaggae K.; Giraldi S.; Campos T. Consenso de cuidado com a pele do recém-nascido. Sociedade Brasileira de Pediatria. 2015. p.1-56. 